\title{
Negative pressure wound therapy: Regulating blood flow perfusion and microvessel maturation through microvascular pericytes
}

\author{
ZHANJUN MA, ZONGHUAN LI, KANGQUAN SHOU, CHAO JIAN, \\ PENGCHENG LI, YAHUI NIU, BAIWEN QI and AIXI YU
}

Zhongnan Hospital of Wuhan University, Department of Orthopedics, Wuhan University, Wuhan, Hubei 430071, P.R. China

Received February 22, 2016; Accepted July 11, 2017

DOI: $10.3892 /$ ijmm.2017.3131

\begin{abstract}
Negative pressure wound therapy (NPWT) has been demonstrated to accelerate wound healing by promoting angiogenesis. However, whether blood flow perfusion is regulated by microvessel maturation and pericytes following NPWT remains unclear, as well as the exact association between pericytes and collagen type IV.The aim of this study was to investigate the relevant association between blood flow perfusion and microvessel maturation and pericytes following NPWT, and to further explore the underlying molecular mechanisms. We also aimed to investigate the association between pericytes and collagen type IV. For this purpose, we created a rat model of diabetic wounds and microvascular blood flow perfusion was detected using a laser Doppler blood perfusion imager. The expression levels of angiogenin-1, tyrosine phosphorylation of tyrosine kinase receptor-2 (Tie-2), $\alpha$-smooth muscle actin ( $\alpha$-SMA) and collagen type IV were detected and analyzed through immunohistochemistry, immunofluorescence, RT-qPCR and western blot analysis. The results revealed that NPWT promoted the overexpression of angiogenin-1, Tie-2, $\alpha$-SMA and collagen type IV, and significantly increased blood flow perfusion coupled with microvessel maturation in the NPWT group at the later stages (7-10 days) of wound healing. Our results suggested that NPWT can preferentially enhance vessel maturation and increase the number of pericytes, thus regulating blood flow perfusion. On the other hand, pericytes and collagen type IV had a mutual interaction, promoting microvessel maturation.
\end{abstract}

Correspondence to: Dr Baiwen Qi or Professor Aixi Yu, Zhongnan Hospital of Wuhan University, Department of Orthopedics, Wuhan University, 169 Donghu Road, Wuhan, Hubei 430071, P.R. China E-mail:wb002162@whu.edu.cn

E-mail: yuaixi@whu.edu.cn

Key words: blood flow perfusion, collagen type IV, negative pressure wound therapy, pericytes, vessel maturation

\section{Introduction}

As a non-invasive wound therapy method, negative pressure wound therapy (NPWT) has been extensively applied to accelerate wound healing in chronic, acute and complex wounds (1). The technique includes negative pressure and an airtight wound, and the suction force created by the NPWT equipment that helps to drain excess fluid, leading to the alliviation of wound edema and bacterial count reduction, thus promoting granulation tissue formation, as well as affecting blood flow perfusion in the wound $(2,3)$.

For accelerating the speed of wound healing, NPWT has mainly been applied to wounds that are open and hard to agglutinate. The relevant molecular mechanisms and biological effects primarily include the following (4,5): macrodeformation, microdeformation, removal of exudate and maintaining a warm and moist microenvironment on the surface of the wound. These molecular mechanisms and biological effects play vital roles in inducing the deformation of wound tissue $(1,6)$; in addition, tissue deformation stimulates wound angiogenesis, the overexpression of growth factors and fresh granulation tissue formation $(7,8)$. On the other hand, NPWT can promote collagen deposition, a vital component of granulation tissue in the wound site, which serves as a critical component of blood vessels and affects wound healing (4). Since the formation of fresh granulation tissue is a critical process of the tissue proliferation stage during wound healing (9), the quality of fresh granulation tissue could determine the rate of wound healing (9).

Although the positive therapeutic effects of NPWT have been generally recognized, its side-effects are not yet fully understood. There are numerous controversies as to the side-effects and the identification of the related molecular mechanisms, particularly in terms of blood flow perfusion following NPWT treatment. Xia et al (10) found that blood flow perfusion was significantly increased on the surface of extremity wounds following NPWT treatment. However, a recent study reported that blood flow perfusion was decreased in the wound following NPWT treatment (11). It is well known that alterations in blood flow can be affected by many factors, and previous studies have mostly focused on the application of physical methods for detecting alterations in blood flow in the 
wound $(11,12)$. Therefore, in the present study, and to the best of our knowledge, for the first time, we investigate and explore alterations in wound blood flow coupled with microvascular characteristics and relevant expression levels on the molecular level using blood flow physical test methods following NPWT treatment.

In the present study, a tyrosine kinase receptor-2 (Tie-2) inhibitor was successfully applied to block vessel maturation and pericyte proliferation. Microvascular blood flow perfusion was detected using a laser Doppler blood perfusion imager, and the expression levels of angiogenin-1 (Ang-1), the tyrosine phosphorylation of Tie-2 (p-Tie-2), $\alpha$-smooth muscle actin ( $\alpha$-SMA) and collagen type IV were detected and analyzed through immunohistochemistry, immunofluorescence, RT-qPCR and western blot analysis. We further discuss whether blood flow perfusion is increased following microvessel maturation and the number of pericytes, and we also further explore the underlying molecular mechanisms. In addition, we investigate the association between pericytes and collagen type IV.

\section{Materials and methods}

Animals. A total of 96 Sprague-Dawley rats with a male:female at a ratio of 1:1, weighing approximately 210-250 g, were obtained from the Experimental Animal Central Research Laboratory at Wuhan University (Wuhan, China). All rats were individually caged under specific pathogen-free conditions, housed under a controlled temperature of approximately $24 \pm 1^{\circ} \mathrm{C}$ and a relative humidity of $45-55 \%$ and a $12-\mathrm{h}$ light/12-h dark cycle. The rats were allowed ad libitum to drink filtered water and eat standard animal feed. All experiments were carried out between 09:00 and 17:00 h. Treatment of the animals was carried out in strict accordance with the recommendations described in the Guide for the Care and Use of Laboratory Animals of the National Institutes of Health. The experimental protocol was approved by the Committee on the Ethics of Animal Experiments of Wuhan University, Wuhan, China. All surgical procedures were performed under chloral hydrate anesthesia, and efforts were made to minimize animal suffering.

Induction and maintenance of diabetes in rats. A total of 96 rats were administered a single injection of freshly dissolved streptozotocin (STZ; 100 mg/kg; Sigma, St. Louis, MO, USA) in a $0.1 \mathrm{mmol} / 1$ citrate buffer $(\mathrm{pH} 4.5)$ intraperitoneally after overnight fasting, as previously described (13). The rat diabetic model was successfully established by detecting the fasting blood glucose levels of the rats (measured using a glucometer; Johnson \& Johnson, Milpitas, CA, USA).

Three days after the STZ injection, the rats were screened for serum glucose levels. The diabetes mellitus status was determined, with continuation to the next step of the experiment if the fasting blood glucose level remained at $>300 \mathrm{mg} / \mathrm{dl}$ for 7 days. All the rat fasting blood glucose levels were detected 3 times a week. Minimal insulin doses of 0.1-0.2 units/mouse were administered by subcutaneous injection if the fasting serum glucose levels of the rats were $>450 \mathrm{mg} / \mathrm{dl}$. The fasting blood glucose levels of the rats were sustained at a level $>300 \mathrm{mg} / \mathrm{dl}$ during the experimental period.
Wound protocol and animal grouping. The dorsa of all the animals were completely shaved with an electrical clipper $24 \mathrm{~h}$ prior to the experiment. General intraperitoneal anesthesia was conducted by injection of $350 \mathrm{mg} / \mathrm{kg}$ chloral hydrate $(7 \%)$ 5 min prior to the surgery. The dorsa of all the rats were disinfected with povidone iodine solution and $75 \%$ medical alcohol, and a $3.5 \times 3.5 \mathrm{~cm}$ area of skin and panniculus carnosus were removed to create a full-thickness diabetic wound, the depth extending to the deep fascia on the dorsum. All animals were randomly divided into the following 4 groups: NPWT, NPWT + Tie-2 inhibitor (NPWT + Tie), gauze and gauze + Tie-2 inhibitor (gauze + Tie). In the NPWT and NPWT + Tie groups, a total of 48 rats with diabetes mellitus wounds were covered with a $3.5 \times 3.5 \mathrm{~cm}$ area of black polyurethane (PU) foam (VSD Medical Technology Co., Ltd., Hubei, China) and then with a vacuum-assisted closure device (Wego, Shandong, China). The negative pressure value was set constantly at continuous $-125 \mathrm{mmHg}$. The VAC device affected neither the ambulation nor the diet and lifestyle of the treated animals (data not shown). In the gauze and gauze + Tie groups, a total of 48 rats with diabetes mellitus wounds received petrolatum gauze treatment. In addition, in the NPWT + Tie and gauze + Tie groups, a total of 48 rats with diabetes mellitus were selected to receive an intraperitoneal injection of $50 \mathrm{mg} /$ kg Tie-2 kinase inhibitor (Selleck Chemicals, Houston, TX, USA) twice a week, which was dissolved into a $1 \mathrm{ml}$ of the vehicle (5\% ethanol, 5\% Cremophor and 90\% distilled water), as previously described (14) and according to the supplier's instructions.

In total, 24 rats were euthanized by cervical dislocation on the 1st, 3rd, 7th and 10th day after the surgery [24 rats (6 from each group) were sacrificed on each selected day]. The wound fresh granulation tissue samples were harvested aseptically from the surface of the wounds. Of the samples that were bisected, half were placed in $4 \%$ neutral formalin formaldehyde (Aspen, Wuhan, China) and the other half were snap-frozen in liquid nitrogen and stored at $-80^{\circ} \mathrm{C}$.

Wound surface blood flow detection. On the 1st, 3rd, 7th and 10th day after the surgery, a laser Doppler blood perfusion imager (LDPI) and PeriScan PIM 3 system (both from Perimed, Stockholm, Sweden) was used to scan the blood flow of the wound surface, as previously described (15). Subsequently, PIM software version 1.5 (Perimed) was applied to analyze blood flow changes on the scanned images, as previously described (10).

Wound contraction analysis. The skin wound site in each rat was digitally photographed at the prescribed time points, and the wound contraction was measured using Image Pro-Plus version 6.0 (Media Cybernetics, Bethesda, MA, USA) under double-blinded conditions. The wound areas of the each rat were normalized to the initial wound size, and the wound healing rate was expressed as a percentage of wound contraction as follows: [(wound area on day 0 - wound area on day $\mathrm{n}$ )/ (wound area on day 0)] x100\% $(n=1,3,7$ and 10). All data were expressed as the means $\pm \mathrm{SD}$, and compared among the different groups using one-way analysis of variance.

Immunohistochemistry. The samples were embedded in paraffin wax and sectioned serially into $5-\mu$ m-thick slices. 
The slices were stained with standard haematoxylin and eosin (H\&E) (Boster Biological Technology, Hubei, China) stain for observation of the histological changes in the wounds.

Antibodies against rat Ang-1 (ab102015; 1:200; Abcam, Cambridge, UK), Tie-2 (sc-9026; 1:200; Santa Cruz Biotechnology, Inc., Dallas, TX, USA), $\alpha$-SMA (ab32575; 1:200) and collagen type IV (ab6586; 1:200) (both from Abcam) served as the primary antibodies. The sections were dewaxed and hydrated for immunohistochemical staining. First, the activity of endogenous peroxidase was quenched with $(3 \%)$ hydrogen peroxide (Aspen). Second, citrate buffer was used for antigen retrieval. The sections were then treated with microwave (Galanz, Guangdong, China) set at $500 \mathrm{~W}$ for $5 \mathrm{~min}$. Third, the sections were incubated with primary antibodies and set at $4^{\circ} \mathrm{C}$ overnight. Fourth, the sections were washed 3 times with phosphate-buffered saline (PBS; Bioyear, Hubei, China), and subsequently the sections were incubated with goat anti-rabbit secondary antibody (1:500; Aspen) for $30 \mathrm{~min}$. Fifth, the sections were incubated with avidin-biotin complex (VECTASTAIN Elite ABC kit; Vector Laboratories, Inc., Burlingame, CA, USA) for $30 \mathrm{~min}$. Finally the reaction was visualised by using 3'3-diaminobenzidine (Dako, Glostrup, Denmark), and with hematoxylin and eosin to stain the nuclei (Sigma-Aldrich). The negative control groups staining experiments were performed using 1:1,000 normal goat serum substituting for primary antibody in each section. The images were captured using a light microscope (BX51WI; Olympus Corp., Tokyo, Japan).

Immunofluorescence staining. A double-labeling immunofluorescence technique was applied to analyze the number of pericytes and the microvascular pericyte coverage index (MPI). The pericyte coverage of the microvessels was detected using anti-CD31 (ab9498; 1:400) and anti- $\alpha$-SMA (ab124964; 1:400) antibodies (both from Abcam). The sections were blocked with BSA $(5 \%)$ for $2 \mathrm{~h}$ and incubated with primary antibody at $4^{\circ} \mathrm{C}$ overnight. After thorough washing, the sections were incubated with secondary goat anti-rabbit secondary antibodies (Ki67, AS1110; CD31, AS1111; $\alpha$-SMA, AS1110; 1:400; all from Aspen) for $1 \mathrm{~h}$ in the dark. Subsequently, the sections were incubated in 4'6-diamidino-2 phenylindole (DAPI; Aspen) for nuclei staining. All images were captured using a fluorescence microscope (Eclipse TE2000-E; Nikon Corp., Tokyo, Japan), and images were merged by using Image Pro-Plus version 6.0 software (Media Cybernetics, Inc., Rockville, MD, USA).

Quantification of microvessel density (MVD). The MVD counting technique has been widely used to assess blood vessel number (16). MVD was quantified as the average number of microvessels per viewing field, and red CD31 staining was used as an endothelial cell marker. CD31-positive endothelial cells and neglected vessels with or without lumen were counted under a power field of 20x10 in 5 randomly selected fields from 3 separate sections of each sample.

Quantification of the MPI. MPI was used to assess the maturity of new blood vessels, as previously described $(17,18)$. MPI was correspondingly established by quantifying the percentage of CD31-positive microvessels that exhibited co-localization of endothelial cell staining (CD31) and pericyte immunostaining ( $\alpha$-SMA) under a power field of $10 \times 20$, as previously described (18). A single endothelial cell was regarded as a unit of quantification regardless of whether it formed a tube, and a pericyte was defined as a single layer of $\alpha$-SMA-positive cells co-localizing with CD31-positive cells. For MPI quantification, at least 5 non-overlapping microscopic fields per section were independently analyzed under doubleblinded conditions. The MPI was expressed as the $\alpha$-SMA/ CD31 ratio.

Quantitative RT-PCR (RT-qPCR). Total RNA was extracted using an RNeasy mini kit (Qiagen AB, Sollentuna, Sweden), and $5 \mu \mathrm{g}$ RNA was reverse transcribed into cDNA using the RevertAid First Strand cDNA Synthesis kit (Fermentas; Thermo Fisher Scientific, Inc., Waltham, MA, USA) to a final reaction volume of $20 \mu \mathrm{l}$ and an S1000 Thermal Cycler (Bio-Rad Laboratories, Inc., Hercules, CA, USA) at $65^{\circ} \mathrm{C}$ for $5 \mathrm{~min}$, cooling on ice and $42^{\circ} \mathrm{C}$ for $60 \mathrm{~min}$, according to the manufacturer's protocol. The reaction was terminated by heating at $70^{\circ} \mathrm{C}$ for $5 \mathrm{~min}$. The primer sequences are as follows: Ang-1 forward, 5'-TAA CCT CGC CCT GCA AAG AG-3' and reverse primer, 5'-CTG TAT GCT TGC AGG TGG TGAT-3'; $\alpha$-SMA forward, 5'-CAA CCC CTA TAC AAC CAT CAC AC-3' and reverse primer, 5'-CCC AAA CTG CTT GCG TAA CC-3'; collagen IV forward, 5'-ACT GTG GAT TGG CTA TTC CTT TG-3' and reverse primer, 5'GCT TCT TGA ACA TCT CGC TTC TC-3'; and glyceraldehyde 3-phosphate dehydrogenase (GAPDH) forward, 5'-CGC TAA CAT CAA ATG GGG TG-3' and reverse primer, 5'-TTG CTG ACA ATC TTG AGG GAG-3'.

Following DNase treatment to remove genomic DNA, RT-qPCR was performed to a final volume of $20 \mu \mathrm{l}$ using $1 \mu \mathrm{l}$ template cDNA, $10 \mu \mathrm{l}$ SYBR qPCR mix (2X; Toyobo Co., Ltd., Osaka, Japan), $6.6 \mu \mathrm{l}$ diethylpyrocarbonate-treated water, $1 \mu \mathrm{l}$ forward primer $(5 \mu \mathrm{m}), 1 \mu \mathrm{l}$ reverse primer $(5 \mu \mathrm{m}), 0.4 \mu \mathrm{l} \mathrm{ROX}$ reference dye (50X) on a iQ5 Real-Time PCR detection system (Bio-Rad Laboratories, Inc.). Respective negative (no cDNA) and RT controls were used for each gene. The thermocycling profile for SYBR-reen RT-qPCR was as follows: initially set at $95^{\circ} \mathrm{C}$ and sustained for $1 \mathrm{~min}$ for the initial denaturation step; followed by 40 cycles of degeneration with the temperature set at $95^{\circ} \mathrm{C}$, which was held for $15 \mathrm{sec}$, then set at $60^{\circ} \mathrm{C}$ and sustained for $15 \mathrm{sec}$ for annealing; and, finally, the elongation step, involving a $60 \mathrm{sec}$ hold at $72^{\circ} \mathrm{C}$. Each sample was run in triplicate and quantified using the $2^{-\Delta \Delta \mathrm{Ct}}$ method to determine relative mRNA expression levels.

Western blot analysis. The samples were homogenized and total proteins extracted using a radioimmunoprecipitation assay (RIPA) buffer (Beyotime Institute of Biotechnology, Haimen, China). A BCA kit (Beyotime Institute of Biotechnology) was used to determine the protein concentration. The proteins were loaded on sodium dodecyl sulfate (SDS) polyacrylamide gels (10\%) (Aspen), transferred onto nitrocellulose membranes (Pall Corp., New York, NY, USA), and blocked in non-fat dry milk $(5 \%)$ at room temperature to set for $2 \mathrm{~h}$. The membranes were incubated overnight at $4^{\circ} \mathrm{C}$ with primary antibodies against Ang-1 (1:1,000; ab102015; Abcam), p-Tie-2 (1:1,500; sc-9026; Santa Cruz Biotechnology, Inc.), $\alpha$-SMA (1:2,000; ab32575), collagen type IV $(1: 1,500$; ab6586) and GAPDH (1:5,000; ab37168) (all from Abcam). 
Subsequently, the membranes were incubated with horseradish peroxidase-conjugated secondary antibody (Aspen) for $1 \mathrm{~h}$, and finally the membranes were detected using an enhanced chemiluminescence substrate (Beyotime Institute of Biotechnology).

Statistical analysis. All data are presented as the means \pm SD and statistical significance was assessed by one-way analysis of variance (ANOVA). SPSS 18.0 software (SPSS, Chicago, IL, USA) was used for statistical analysis. Differences between groups were considered statistically significant at $\mathrm{P}<0.05$ or $\mathrm{P}<0.01$

\section{Results}

Wound contraction. The wound contraction was not markedly altered in the early stages (1-3 days) of wound healing in the 4 groups, although a small number of granulation tissue implants in the wound was observed in the NPWT group on the 3rd day; however, there was an abundance of fresh granulation tissues filled in the surface area of the wound accompanied by marked wound contraction in the NPWT group on the 7 th day (Fig. 1A). In the other groups, less granulation tissue was found in the wounds, particularly in the NPWT + Tie and gauze + Tie groups on the 7 th day. The percentage of wound contraction in the NPWT group was significantly higher than those in the other groups at day 7 and $10(\mathrm{P}<0.05)$ (Fig. 1B). On the 10th day, wound contraction was significantly greater in the NPWT group than in the other groups $(\mathrm{P}<0.05$ for the gauze group, $\mathrm{P}<0.01$ for the NPWT + Tie group or gauze + Tie group) (Fig. 1B).

Changes in microvessel blood flow perfusion. Wound blood flow perfusion is shown in Fig. 1C. The results revealed that the areas of blood flow perfusion were gradually extended and enlarged in the NPWT group from the 3rd to the 10th day. However, there were fewer areas of blood perfusion in the gauze group during that time period. The wound blood flow perfusion value gradually increased from the 3 rd to the 10th day in the NPWT group and gauze group, and it was significantly higher in the NPWT group than in the gauze group during that time period $(\mathrm{P}<0.05$; Fig. 1D). In the NPWT + Tie and gauze + Tie groups, the area of blood flow perfusion was the least among all the groups, and the blood flow perfusion value was also the lowest among all the groups, with the difference being statistically significant compared to that in the NPWT group from the 7 th to the 10th day $(\mathrm{P}<0.01 ;$ Fig. 1D).

Histopathological analysis. In the early stages, in the NPWT group, the number of new blood vessels was higher than that in the other groups, and the degrees of inflammatory cell infiltration and epidermal necrolysis were more slight in the NPWT group than in the other groups. On the 7th and 10th day, in the NPWT group, the new microvessels were abundant, and the collagen fibrils were distributed compactly and regularly; by contrast, compared to the NPWT group, the lower number of new blood vessels in the NPWT + Tie and gauze + Tie groups, and the collagen fibrils were broken (Fig. 2).

MVD. The double immunofluorescence marker, CD31, and $\alpha$-SMA were combined to mark vascular endothelial cells and microvascular pericytes (Fig. 3). On the 1st day, a few CD31-positive cells were observed in the NPWT group, and a small amount of endothelial cells was detected with red marker CD31-positive cells in the NPWT group at day 3 (data not shown). On the 7th day, a large amount of endothelial cells was detected; in addition, a small number of endothelial cells was observed in the gauze group on the $3 \mathrm{rd}$ and 7 th day, although it was less than that in the NPWT group. the In NPWT + Tie and gauze + Tie groups, the number of endothelial cells with red marked CD31-positive cells was less than that in the gauze group. The MVD was used to quantify the number of new blood vessels, and the results revealed that the MVD was higher in the NPWT group than the other groups from day 1 to day $10(\mathrm{P}<0.05$ for gauze group; $\mathrm{P}<0.01$ either NPWT + Tie group or gauze + Tie group) (Fig. 4A.)

The number of pericytes and MPI. We used a combination of the specific markers, CD31 and $\alpha$-SMA, to simultaneously immunostain microvascular endothelial cells and pericytes to assess the functional status of the neovasculature in the wound (19). The results revealed that a small number of green marker $\alpha$-SMA-positive pericytes was detected on the 3rd day in the NPWT group and gauze group. By contrast, in the other groups, few pericytes were detected on the 3rd day (data not shown). On the 7th day, a great number of pericytes was detected, and was distributed discontinuously around the endothelial cell lumen in the NPWT group. An abundance of pericytes was detected in the NPWT group on the 10th day, with the pericytes tightly encircling and covering the microvessel endothelial tubes (Fig. 3). Subsequently, a small number of pericytes was detected in the gauze group on the 3rd and 7th day, and a number of pericytes was detected on the 10th day in the gauze group; however, this number was significantly lower than that in the NPWT group at the same time points $(\mathrm{P}<0.05)$, and in the NPWT group it was significantly higher than that in the NPWT + Tie group or gauze + Tie group $(\mathrm{P}<0.01$; Fig. 4B).

Although the MVD values can be applied to evaluate the presence of new blood vessels, these MVD values do not provide the information of the functional status of the microvessels or the maturity of the neovasculature (18). Therefore, the MPI values were applied to reflect the percentage of microvessels covered with pericytes and quantified to assess the maturity of the neovasculature (20). The results revealed that in the NPWT group, a significantly higher average MPI was detected compared to the gauze group from the 3rd to the 10th day $(\mathrm{P}<0.05)$, and the MPI was significantly higher than that in the Tie-2 inhibitor groups $(\mathrm{P}<0.01$; Fig. 4C).

Changes in the expression of Ang-1 and p-Tie-2 following NPWT treatment. The changes in the mRNA expression levels of Ang-1 were quantitatively analyzed by RT-qPCR, as shown in Fig. 5A. In the NPWT group, the mRNA expression of Ang-1 was significantly lower than that in the other groups from the 1 st to the 3 rd day $(\mathrm{P}<0.05$ vs. gauze group; $\mathrm{P}<0.01$ vs. either NPWT + Tie group or gauze + Tie group). However, on the 7th and 10th day, the mRNA expression of Ang-1 was significantly higher than that in the other groups $(\mathrm{P}<0.05$ vs. gauze group; $\mathrm{P}<0.01 \mathrm{vs}$. either NPWT + Tie group or gauze + Tie group). In the NPWT + Tie and gauze + Tie groups, the mRNA 

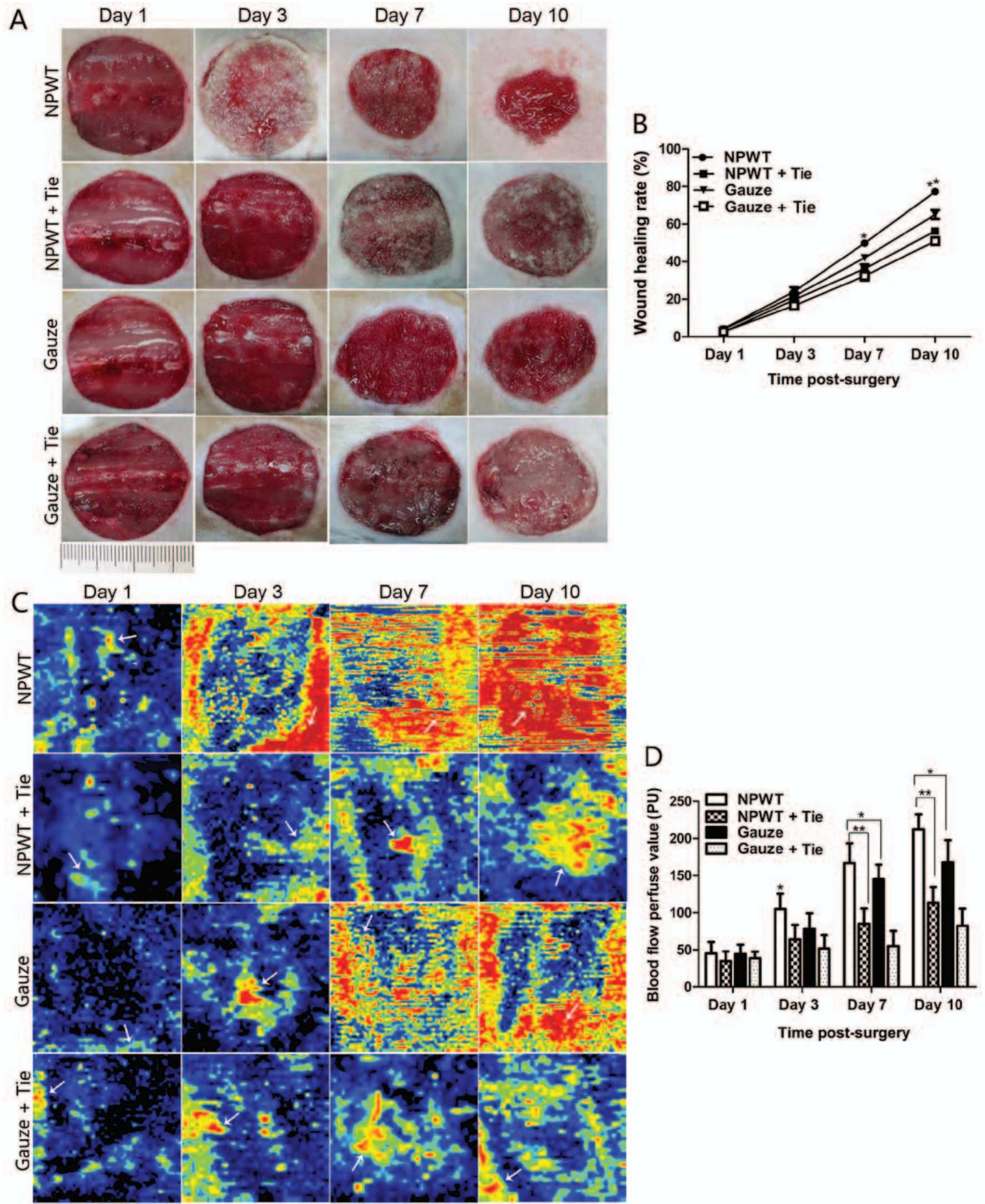

Figure 1. Wound healing rate and changes in blood flow in the different groups. (A) The macroscopic wound healing process in the different groups. (B) Statistical analysis of wound healing rate in the 4 groups. (C) The changes in blood flow perfusion in the different groups during wound healing. The areas of yellow and red in the image represent the quantity of blood flow in the wound (white arrow), as well as the areas of blue imply less or no blood flow (white arrow). (D) Statistical analysis of blood flow perfusion value in the different groups. All data are expressed as the means $\pm \mathrm{SD}\left({ }^{*} \mathrm{P}<0.05\right.$ and $\left.{ }^{* *} \mathrm{P}<0.01\right)$.

expression trend of Ang-1 was opposite to that in the NWPT group, and the mRNA expression of Ang-1 was significantly lower than that in the NPWT group on days 7 and $10(\mathrm{P}<0.01)$. Representative western blots of Ang-1 protein expression are 


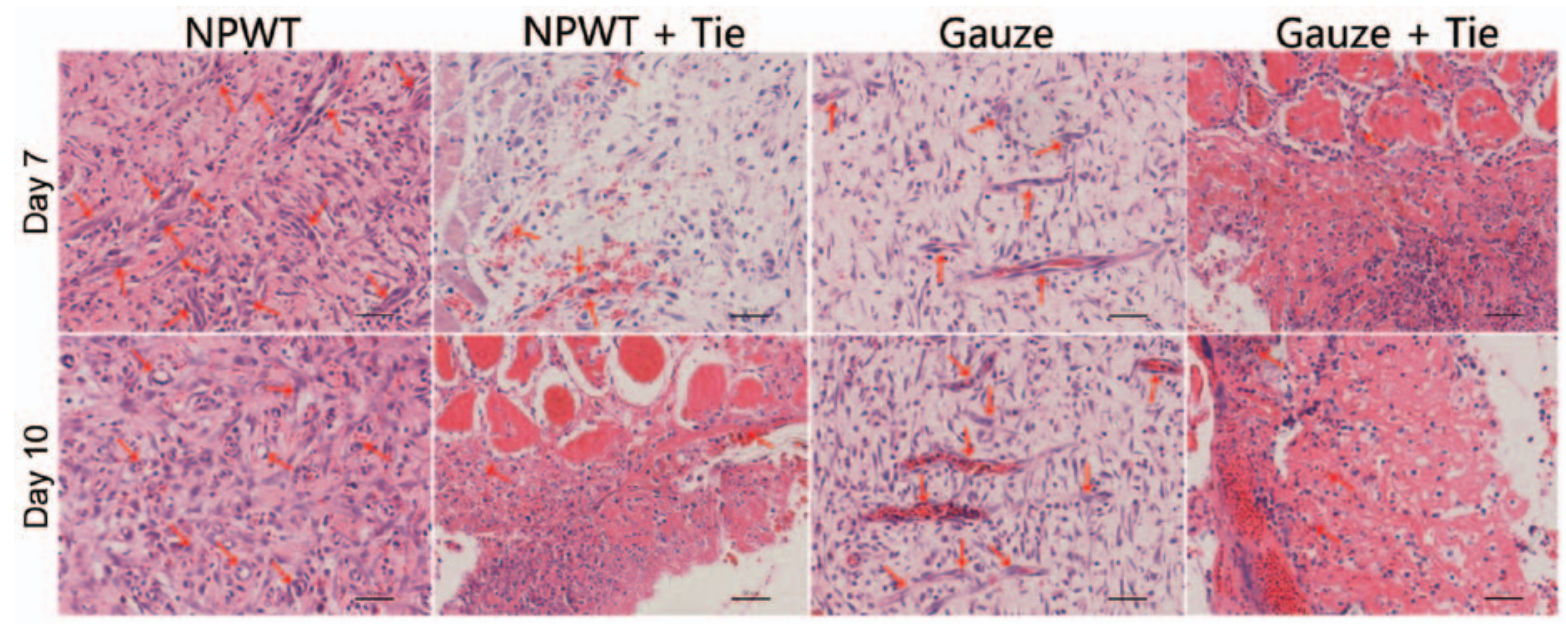

Figure 2. Histological changes in the different groups. On days 7 and 10, abundant granulation tissue and new microvessels filling the wound in the negative pressure wound therapy (NPWT) group (red arrows) were observed; however a small amount of granulation tissue and new blood vessels were observed in the other groups (red arrow). Scale bar, $50 \mu \mathrm{m}$.

shown in Fig. 5B, which present the same trend as Ang-1 mRNA expression. The results of statistical analysis of Ang-1 protein expression are shown in Fig. 5C. Immunohistochemical staining also revealed that Ang-1 was primarily presented in pericytes, and in the NPWT group, Ang-1-positive staining was much higher than that in the other groups on the 7th day (Fig. 5D).

The tyrosine phosphorylation of Tie- 2 was detected by western blot analysis as shown in Fig. 5B. The results revealed that the expression of p-Tie-2 was gradually decreased from days 1 to 3 in the NPWT group, and it was significantly lower than that in the other groups at the same time points $(\mathrm{P}<0.05$ vs. gauze group; $\mathrm{P}<0.01$ vs. either NPWT + Tie group or gauze + Tie group); subsequently, it increased rapidly from days 7 to 10 in the NPWT group, and was significantly higher than that in the other groups $(\mathrm{P}<0.05$ vs. gauze group; $\mathrm{P}<0.01$ vs. either NPWT + Tie group or gauze + Tie group) (Fig. 5E). Although the expression trend of p-Tie-2 in the gauze group was similar to that in the NPWT group, the expression level of p-Tie-2 was significantly lower than that in the NPWT group on days 7 and $10(\mathrm{P}<0.05)$. The expression level of p-Tie-2 was gradually decreased in the NPWT + Tie and gauze + Tie groups, and it was significantly lower than that in the NPWT group from the 3rd to the 10th day $(\mathrm{P}<0.01)$. Immunohistochemical staining revealed that Tie-2 was mainly expressed in endothelial cells, and in the NPWT group, Tie-2 positive staining was much higher than that in the other groups on the 7 th day (Fig. 5F).

Changes in the expression levels of $\alpha$-SMA and collagen type IV following NPWT treatment. Changes in the mRNA expression levels of $\alpha$-SMA are shown in Fig. 6A. The mRNA expression of $\alpha$-SMA gradually increased from days 3 to 10 in the NPWT group, and it was significantly higher than that in the gauze group $(\mathrm{P}<0.05)$. In particular, in the NPWT + Tie and gauze + Tie groups, the mRNA expression levels of $\alpha$-SMA were significantly lower than those in the NPWT group from the 7 th to the 10 th day $(\mathrm{P}<0.01)$. The protein expression of $\alpha$-SMA was consistent with its mRNA expression profile (Fig. 6B and C). Immunohistochemical staining revealed that $\alpha$-SMA was primarily present in pericytes, and in the NPWT group, $\alpha$-SMA-positive staining was much higher than that in the other groups on the 7th day (Fig. 6D).

Changes in the mRNA expression of collagen type IV are shown in Fig. 6E. The results revealed that the mRNA expression of collagen type IV was gradually increased from the 1st to the 10th day in the NPWT group, and was significantly higher than that in the other groups $(\mathrm{P}<0.05$ vs. gauze group; $\mathrm{P}<0.01$ vs. either NPWT + Tie group or gauze + Tie group), particularly on the 7 th and 10th day. The changes in the protein expression levels of collagen type IV were consistent with its mRNA expression profile. The results are shown in Fig. 6B and F. Immunohistochemical staining revealed that the positive expression level of collagen type IV was higher than that in the other groups on the 7th day (Fig. 6G).

\section{Discussion}

It has been demonstrated that NPWT can promote the overexpression of various cytokines and growth factors (3), as well as improve the microenvironment of wounds, and thus accelerates wound healing $(21,22)$. In this study, we found that NPWT not only increased microvascular density, but also promoted microvascular stabilization and maturation in the wounds, as well as effect blood flow perfusion. To further elucidate the mechansisms through which NPWT mediates blood flow perfusion, we examined and analyzed changes in blood flow perfusion from the aspect of microvessel structure characteristics and vessel maturation, simultaneously, with the addition of physical methods for detecting changes in blood flow following NPWT treatment.

Ang-1 is primarily expressed in pericyte-like perivascular mural cells (pericytes), suggesting a paracrine effect on new vascular function $(16,23)$, and it serves as a vascular pro-maturation factor that facilitates vascular maturation. Tyrosine kinase receptor Tie-2 can affect vascular maturation and stabilization (24), and it plays a direct role in affecting pericyte recruitment to endothelial cell tubes (23). Ang-1 can bind to Tie-2, and can thus promote microvascular integrity and maturation $(25,26)$. Blocking of Tie-2 leads to tissue edema and hemorrhaging, and retards the vessel remodeling 


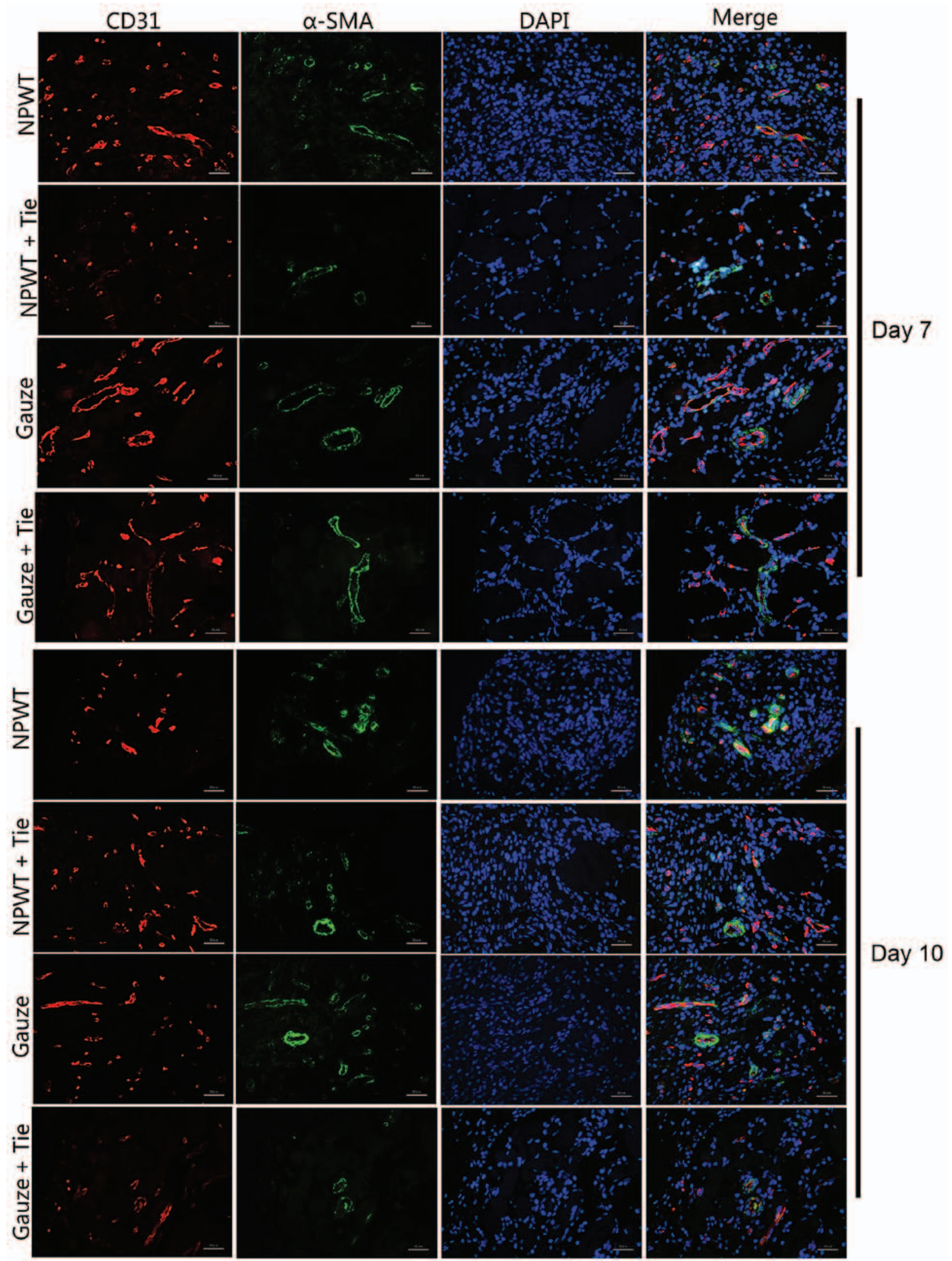

Figure 3. Immunofluorescence staining in the wound. Typical immunofluorescence staining for endothelial cells with CD31 expression (red) and pericytes with $\alpha$-smooth muscle actin ( $\alpha$-SMA) expression (green) on days 7 and 10. Scale bar, $50 \mu \mathrm{m}$. Original magnification, $\mathrm{x} 200$.

and maturation process (23). Based on these findings, in this study, we found that the levels of Ang-1 and p-Tie-2 were preferentially and coordinately increased or decreased in the
NPWT group; the results suggested that Ang-1 preferentially binds to Tie-2 and promotes phosphorylation only following NPWT treatment, and thus coordinately helps to complete 

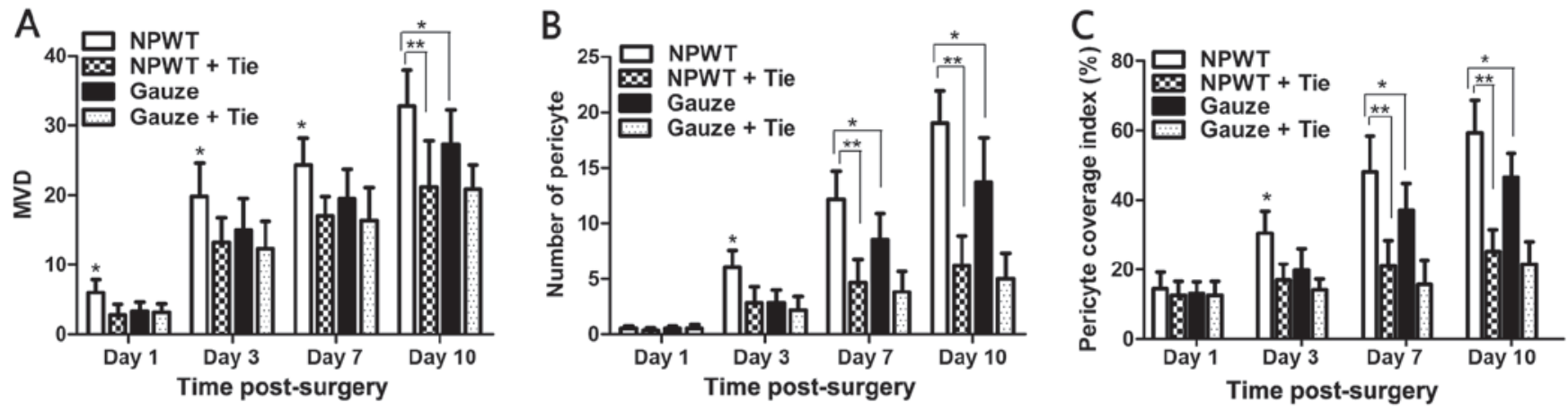

Figure 4. Statistical analysis of microvessel density (MVD) and microvascular pericyte coverage index (MPI). (A) The MVD at different time points and in the different groups. (B) The number of pericytes in the 4 groups during wound healing. (C) The microvascular pericyte coverage index (MPI) was quantified by assessing the percentage of microvessels that were associated with $\alpha$-smooth muscle actin ( $\alpha$-SMA)-positive pericytes. All data are expressed as the means \pm SD $\left(\right.$ P $\mathrm{P}<0.05$ and $\left.{ }^{* *} \mathrm{P}<0.01\right)$
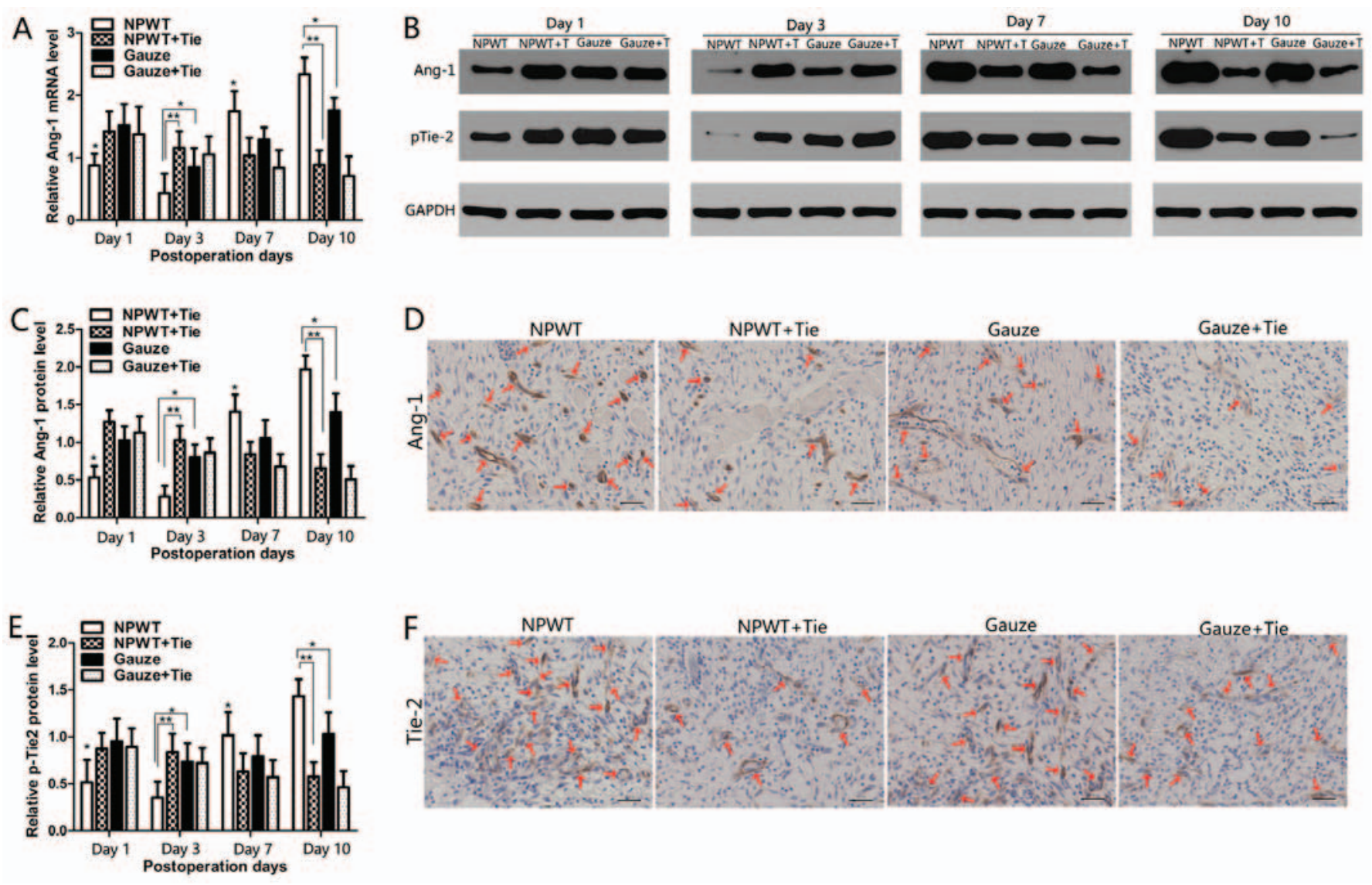

Figure 5. Changes in the expression of angiogenin-1 (Ang-1) and the phosphorylation of tyrosine kinase receptor-2 (p-Tie-2). (A) Gene expression levels of Ang-1 were assessed by RT-qPCR. (B) Representative western blots showing the protein expression of Ang-1 in the different groups. Representative western blots showing Tie2 tyrosine phosphorylation levels in the different groups. (C) Statistical analysis of Ang-1 protein expression levels. (D) Immunohistochemical staining of Ang-1 protein on day 7 after the surgery. The Ang-1 protein signals were mainly present in pericyte-like perivascular mural cells (red arrows) (E) Quantify analysis the expression of p-Tie-2 in the different groups. (F) Immunohistochemical staining of Tie-2 in the different groups on day 7 . Tie-2 protein signals were mainly present in endothelial cells (red arrows). Scale bar, $50 \mu \mathrm{m}$. All data are expressed as the means $\pm \mathrm{SD}\left({ }^{*} \mathrm{P}<0.05\right.$ and $\left.{ }^{* *} \mathrm{P}<0.01\right)$.

microvascular maturation and integrity process. In addition, the levels of Ang-1 and MPI were gradually increased in the NPWT group at the later stages. The results suggested that the microvessels became gradually more mature at the later stages of wound healing following NPWT treatment.

Mature microvessels are usually characterized by the endothelial lumen covered with abundant pericytes, separated by the integral basement membrane (20). In particular, the peri- cyte is an important component of the mature blood vessel (24). Pericytes are ubiquitously present and comprised of a heterogeneous cell population in close contact with endothelial cells. Unlike endothelial cells, pericytes do not form the continuous sheath or directly to the luminal surface of microvessle network. However, pericytes serve as single cells distributed at the capillary network and around blood capillaries, sharing the basement membrane with endothelial cells (27), and they are 

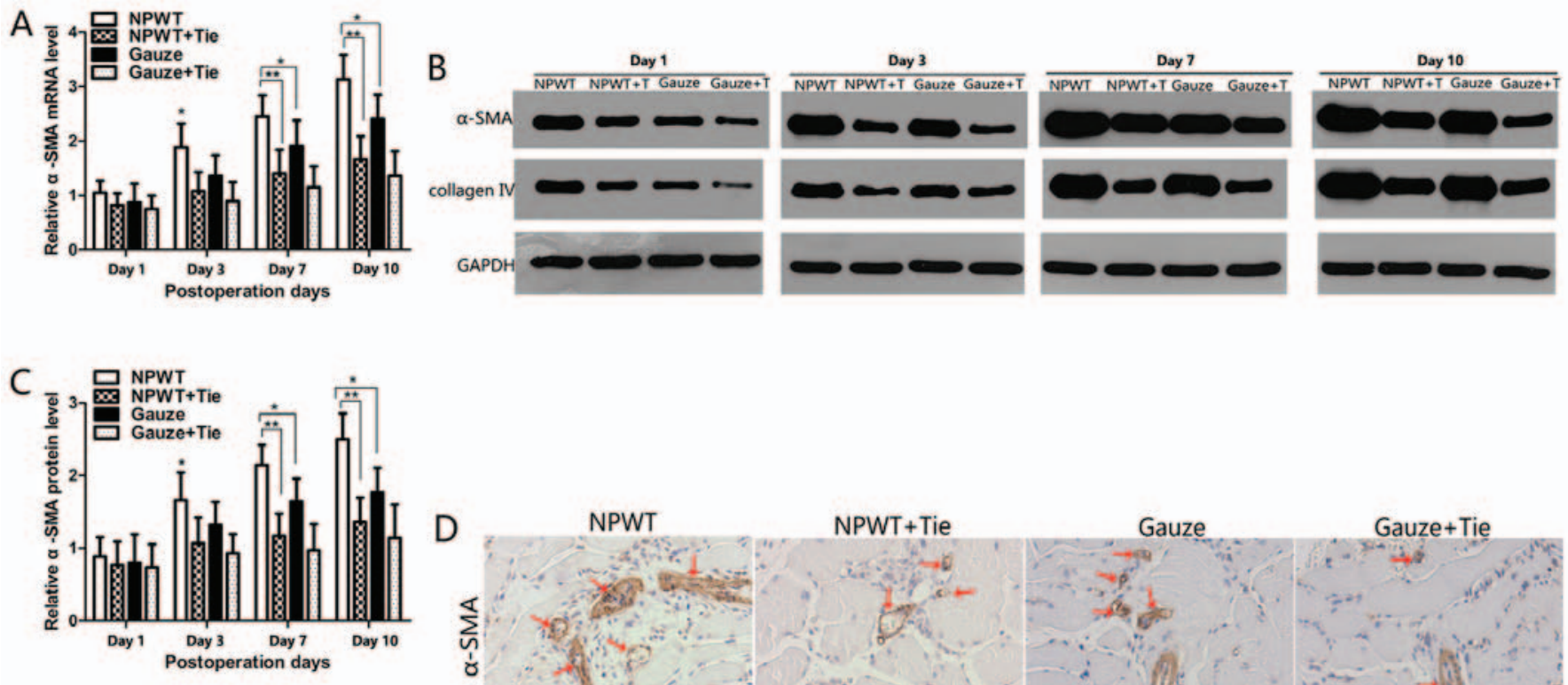

$\mathrm{E}$
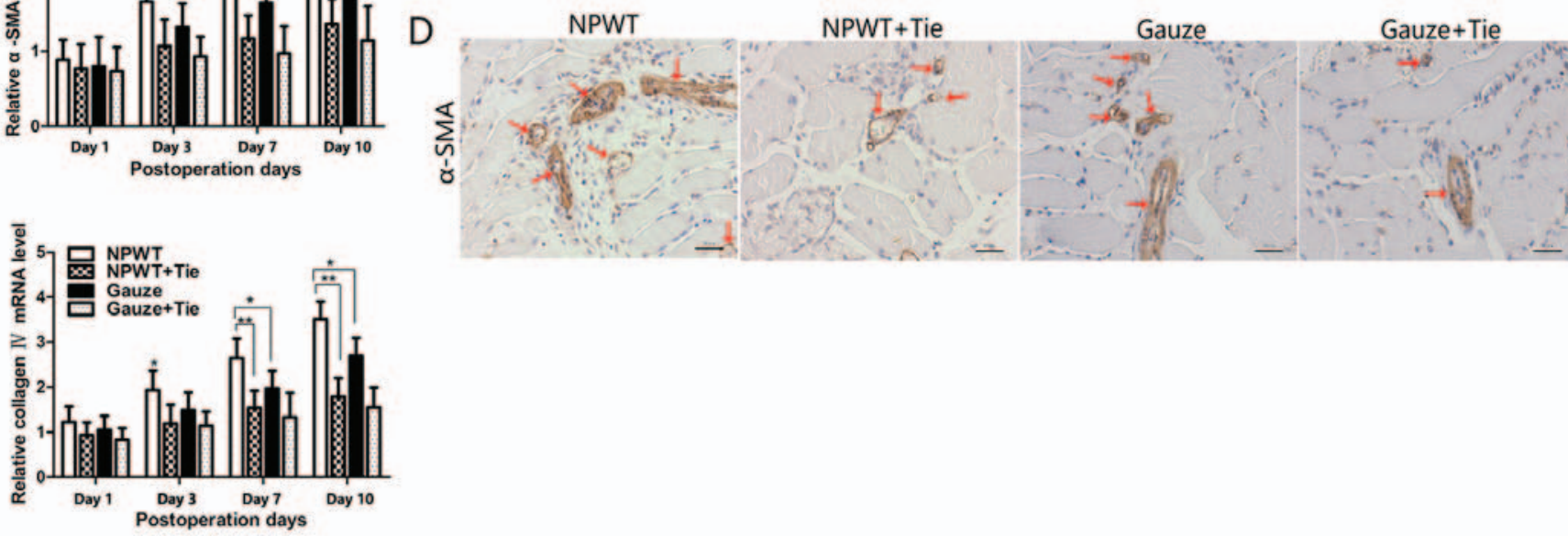

$\mathrm{F}$
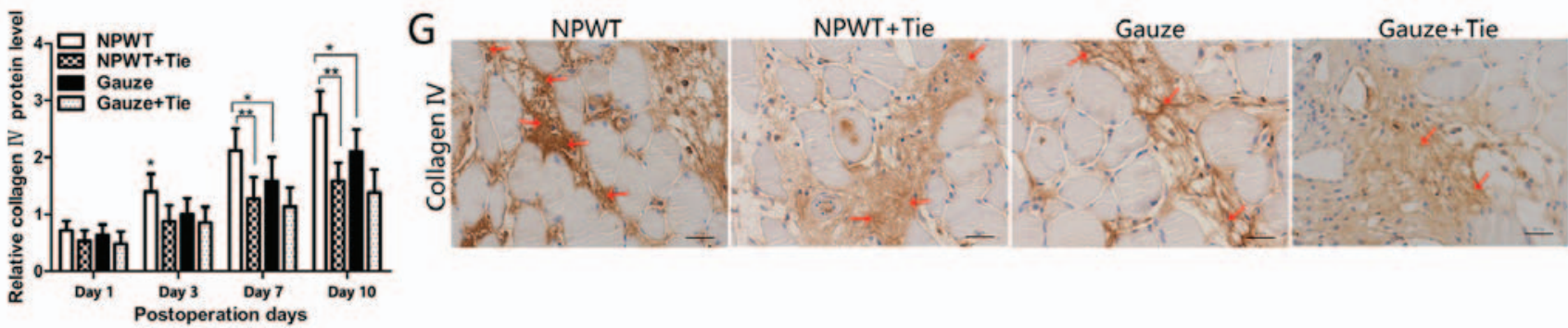

Figure 6. Changes in the expression levels of $\alpha$-smooth muscle actin ( $\alpha$-SMA) and collagen type IV. (A) The expression levels of $\alpha$-SMA mRNA in the different groups. (B) Representative western blots showing the protein expression of $\alpha$-SMA. Representative western blots showing the protein expression of collagen type IV. (C) Quantitative analysis of the protein expression levels of $\alpha$-SMA. The protein expression of $\alpha$-SMA was consistent with its mRNA expression profile. (D) Immunohistochemical staining of $\alpha$-SMA showing that the $\alpha$-SMA protein was mainly present in pericytes (red arrows). (E) Expression levels of collagen type IV gene in the different groups and time points. (F) Quantitative analysis of the protein expression of collagen type IV. (G) Immunohistochemical staining of collagen type IV showing that the positive collagen type IV on day 7, and the positive collagen type IV was higher in the negative pressure wound therapy (NPWT) group than in the other groups (red arrows). Scale bar, $50 \mu \mathrm{m}$. All data are expressed as the means $\pm \mathrm{SD}\left({ }^{*} \mathrm{P}<0.05\right.$ and $\left.{ }^{* *} \mathrm{P}<0.01\right)$.

physically embedded in the basement membrane between the extracellular environment and microvessel tube (28). Previous studies have demonstrated that pericytes have contraction and dilation characteristics on capillaries $(3,24)$, and can regulate cerebral blood flow perfusion $(28,29)$. Studies have also suggested that capillary constriction and dilation are regulated by the vasoactive molecules and neurotransmitters in vitro $(24,30,31)$. In vivo studies have demonstrated that active loss of pericytes will promote capillaries to dilate $(29,32,33)$. Simultaneously, $\alpha$-SMA can serve as a general marker of pericytes $(34,35)$. Therefore a mutual association exists between pericytes and blood flow perfusion, and the pericyte tone may reflect microvascular blood blow perfusion heterogeneity differences (36). In this study, the levels of Ang-1 and $\alpha$-SMA, and MPI were significantly higher in the NPWT group than in the other groups at the later stages. The results suggested that microvessels were preferentially mature in the NPWT group. Simultaneously, pericytes are an important characteristic of mature blood vessels $(16,20,23)$. As shown by our results, pericytes were markedly evident in the NPWT group. Previous studies have suggested that NPWT can promote angiogenesis and enhance wound healing due to the microdeformation and macrodeforation of the wounds (5,37). In this study, we also speculated that microvessel maturation and pericyte proliferation induced by NPWT were closely related to microdeformation and macrodeforation. Unlike the normal microvasculature, tumor blood vessels are tortuous, leaky and show abnormal pericyte coverage, and thus effect blood flow 
perfusion $(38,39)$. We observed that the changes in blood flow perfusion in the whole process of wound healing were affected along with the expression levels of $\alpha$-SMA and MPI in the 4 groups. These results demonstrated that NPWT preferentially promoted the involvement of pericytes, leading to microvessels gradual maturation, and thus changing blood flow perfusion due to the contraction and dilation characteristics of pericytes.

The basement membrane and pericytes serve as two important components of mature blood vessels to affect the microvascular maturation process. Recent studies have suggested that pericytes are recruited to the endothelial cell tube surface, resulting in the deposition of a continuous basement membrane matrix. The endothelial tube is not able to deposit basement membrane in the absence of pericyte $(40,41)$. Collagen type IV serves as a major basement membrane component that can stabilize vascular structure $(42,43)$, regulating vessel morphogenesis and vascular maturation (44). Vessel basement membrane matrix assembly represents a fundamental step in the maturation process of vessels (45). In this study, the expression of collagen type IV gradually increased, and the basement membrane was gradually integrated along with the increase of the number of pericytes in the NPWT group, but in NPWT + Tie and gauze + Tie groups the collagen type IV was markedly reduced and broken, along with a decreased number of pericytes. These results suggested that NPWT preferentially induced pericyte proliferation and basement membrane assembly to form an enveloped endothelium tube, eventually making the new vessels prone to integrity and maturation. Therefore, we demonstrated that pericytes and collagen type IV were complementary to each other for promoting new blood vessel maturation. The results also suggested that the Ang/ Tie-2 signaling pathway not only regulates pericytes, but also regulates basement membrane matrix deposition, assembly and formation. On the other hand, the basement membrane matrix protein deposition and assembly are regulated by the pericytes, even though the molecular mechanisms involved are not yet clear. Tahajjodi et al (43) considered that this process was probably associated with the integrins. In this study, the results demonstrated that pericytes and collagen type IV were complementary to each other for promoting the vascular maturation process jointly.

In conclusion, we demonstrate that NPWT can preferentially promote vessel maturation, and can thus affect blood flow perfusion on the surface of wounds. In particular, pericytes plays a vital role that can effectively affect blood flow in the later stage of wound healing following NPWT treatment. In addition, pericytes and collagen type IV serve as important structural components that are characteristic of vessel maturation, and they complement each other, promoting the maturation of new blood vessels, and thus leading to increased blood flow perfusion on the surface of wounds.

\section{Acknowledgements}

This study was supported by the National Natural Science Foundation of China (no. 81572163) and by the Hubei National Natural Science Fund projects (no. 2014CFB751). We would like to acknowledge the Wuhan VSD Medical Science and Technology, Co., Ltd. (Wuhan, China) for supplying the vacuum material. We would also like to thank the Medical Science
Experimentation Center of Wuhan University providing the experiment equipment. This study has been satisfactorily edited for proper English by an agent of the English Editing Service of Experimental Biology and Medicine.

\section{References}

1. Morykwas MJ, Argenta LC, Shelton-Brown EI and McGuirt W: Vacuum-assisted closure: a new method for wound control and treatment: animal studies and basic foundation. Ann Plast Surg 38: 553-562, 1997.

2. Timmers MS, Le Cessie S, Banwell P and Jukema GN: The effects of varying degrees of pressure delivered by negative-pressure wound therapy on skin perfusion. Ann Plast Surg 55: 665-671, 2005.

3. Li X, Liu J, Liu Y, Hu X, Dong M, Wang H and Hu D: Negative pressure wound therapy accelerates rats diabetic wound by promoting agenesis. Int J Clin Exp Med 8: 3506-3513, 2015.

4. Erba P, Ogawa R, Ackermann M, Adini A, Miele LF, Dastouri P, Helm D, Mentzer SJ, D'Amato RJ, Murphy GF, et al: Angiogenesis in wounds treated by microdeformational wound therapy. Ann Surg 253: 402-409, 2011.

5. Orgill DP and Bayer LR: Negative pressure wound therapy: Past, present and future. Int Wound J 10 (Suppl 1): 15-19, 2013.

6. Morykwas MJ, Simpson J, Punger K, Argenta A, Kremers L and Argenta J: Vacuum-assisted closure: State of basic research and physiologic foundation. Plast Reconstr Surg 117 (Suppl): $121 \mathrm{~S}-126 \mathrm{~S}, 2006$.

7. Chen SZ, Li J, Li XY and Xu LS: Effects of vacuum-assisted closure on wound microcirculation: an experimental study. Asian J Surg 28: 211-217, 2005.

8. Wackenfors A, Sjögren J, Gustafsson R, Algotsson L, Ingemansson $\mathrm{R}$ and Malmsjö M: Effects of vacuum-assisted closure therapy on inguinal wound edge microvascular blood flow. Wound Repair Regen 12: 600-606, 2004.

9. Saxena V, Hwang CW, Huang S, Eichbaum Q, Ingber D and Orgill DP: Vacuum-assisted closure: microdeformations of wounds and cell proliferation. Plast Reconstr Surg 114: 1086-1098, 2004.

10. Xia CY, Yu AX, Qi B, Zhou M, Li ZH and Wang WY: Analysis of blood flow and local expression of angiogenesis associated growth factors in infected wounds treated with negative pressure wound therapy. Mol Med Rep 9: 1749-1754, 2014.

11. Kairinos N, McKune A, Solomons M, Hudson DA and Kahn D: The flaws of laser Doppler in negative-pressure wound therapy research. Wound Repair Regen 22: 424-429, 2014.

12. Lindstedt $\mathrm{S}$, Malmsjö $\mathbf{M}$, Hlebowicz $\mathrm{J}$ and Ingemansson R: Comparative study of the microvascular blood flow in the intestinal wall, wound contraction and fluid evacuation during negative pressure wound therapy in laparostomy using the V.A.C. abdominal dressing and the ABThera open abdomen negative pressure therapy system. Int Wound J 12: 83-88, 2015.

13. Ashokkumar N and Pari L: Effect of N-benzoyl-D-phenylalanine and metformin on carbohydrate metabolic enzymes in neonatal streptozotocin diabetic rats. Clin Chim Acta 351: 105-113, 2005.

14. Hasenstein JR, Kasmerchak K, Buehler D, Hafez GR, Cleary K, Moody JS and Kozak KR: Efficacy of Tie2 receptor antagonism in angiosarcoma. Neoplasia 14: 131-140, 2012.

15. Lindgren M, Malmqvist LA, Sjöberg F and Ek AC: Altered skin blood perfusion in areas with non blanchable erythema: An explorative study. Int Wound J 3: 215-223, 2006.

16. Weidner N: Tumoural vascularity as a prognostic factor in cancer patients: The evidence continues to grow. J Pathol 184: 119-122, 1998.

17. Uemura A, Ogawa M, Hirashima M, Fujiwara T, Koyama S, Takagi H, Honda Y, Wiegand SJ, Yancopoulos GD and Nishikawa S: Recombinant angiopoietin-1 restores higher-order architecture of growing blood vessels in mice in the absence of mural cells. J Clin Invest 110: 1619-1628, 2002

18. Eberhard A, Kahlert S, Goede V, Hemmerlein B, Plate KH and Augustin HG: Heterogeneity of angiogenesis and blood vessel maturation in human tumors: Implications for antiangiogenic tumor therapies. Cancer Res 60: 1388-1393, 2000.

19. Goede V, Schmidt T, Kimmina S, Kozian D and Augustin HG: Analysis of blood vessel maturation processes during cyclic ovarian angiogenesis. Lab Invest 78: 1385-1394, 1998.

20. Zhao J, Chen L, Shu B, Tang J, Zhang L, Xie J, Qi S and Xu Y: Granulocyte/macrophage colony-stimulating factor influences angiogenesis by regulating the coordinated expression of VEGF and the Ang/Tie system. PLoS One 9: e92691, 2014. 
21. Glass GE, Murphy GF, Esmaeili A, Lai LM and Nanchahal J: Systematic review of molecular mechanism of action of negativepressure wound therapy. Br J Surg 101: 1627-1636, 2014.

22. Dumville JC, Hinchliffe RJ, Cullum N, Game F, Stubbs N, Sweeting M and Peinemann F: Negative pressure wound therapy for treating foot wounds in people with diabetes mellitus. Cochrane Database Syst Rev 10: CD010318, 2013.

23. Gaengel K, Genové G, Armulik A and Betsholtz C Endothelial-mural cell signaling in vascular development and angiogenesis. Arterioscler Thromb Vasc Biol 29: 630-638, 2009.

24. Armulik A, Genové G and Betsholtz C: Pericytes: Developmental, physiological, and pathological perspectives, problems, and promises. Dev Cell 21: 193-215, 2011.

25. Kim KE, Cho CH, Kim HZ, Baluk P, McDonald DM and Koh GY: In vivo actions of angiopoietins on quiescent and remodeling blood and lymphatic vessels in mouse airways and skin. Arterioscler Thromb Vasc Biol 27: 564-570, 2007.

26. Holash J, Wiegand SJ and Yancopoulos GD: New model of tumor angiogenesis: Dynamic balance between vessel regression and growth mediated by angiopoietins and VEGF. Oncogene 18: 5356-5362, 1999.

27. Chantrain CF, Henriet P, Jodele S, Emonard H, Feron O, Courtoy PJ, DeClerck YA and Marbaix E: Mechanisms of pericyte recruitment in tumour angiogenesis: A new role for metalloproteinases. Eur J Cancer 42: 310-318, 2006.

28. Kutcher ME and Herman IM: The pericyte: Cellular regulator of microvascular blood flow. Microvasc Res 77: 235-246, 2009

29. Hall CN, Reynell C, Gesslein B, Hamilton NB, Mishra A, Sutherland BA, O'Farrell FM, Buchan AM, Lauritzen M and Attwell D: Capillary pericytes regulate cerebral blood flow in health and disease. Nature 508: 55-60, 2014.

30. Attwell D, Buchan AM, Charpak S, Lauritzen M, Macvicar BA and Newman EA: Glial and neuronal control of brain blood flow. Nature 468: 232-243, 2010.

31. Hamilton NB, Attwell D and Hall CN: Pericyte-mediated regulation of capillary diameter: A component of neurovascular coupling in health and disease. Front Neuroenergetics 2: 2, 2010

32. Peppiatt CM, Howarth C, Mobbs P and Attwell D: Bidirectional control of CNS capillary diameter by pericytes. Nature 443: 700-704, 2006.

33. Puro DG: Physiology and pathobiology of the pericyte-containing retinal microvasculature: New developments. Microcirculation 14: $1-10,2007$.
34. Hellström M, Kalén M, Lindahl P, Abramsson A and Betsholtz C: Role of PDGF-B and PDGFR-beta in recruitment of vascular smooth muscle cells and pericytes during embryonic blood vessel formation in the mouse. Development 126: 3047-3055, 1999.

35. Ohlsson R, Falck P, Hellström M, Lindahl P, Boström H, Franklin G, Ahrlund-Richter L, Pollard J, Soriano P and Betsholtz C: PDGFB regulates the development of the labyrinthine layer of the mouse fetal placenta. Dev Biol 212: 124-136, 1999.

36. Jespersen $\mathrm{SN}$ and Østergaard L: The roles of cerebral blood flow, capillary transit time heterogeneity, and oxygen tension in brain oxygenation and metabolism. J Cereb Blood Flow Metab 32: 264-277, 2012

37. Kim PJ, Attinger CE, Steinberg JS and Evans KK: Negative pressure wound therapy with instillation: Past, present, and future. Surg Technol Int 26: 51-56, 2015.

38. Benjamin LE, Golijanin D, Itin A, Pode D and Keshet E: Selective ablation of immature blood vessels in established human tumors follows vascular endothelial growth factor withdrawal. J Clin Invest 103: 159-165, 1999.

39. Rivera LB and Brekken RA: SPARC promotes pericyte recruitment via inhibition of endoglin-dependent TGF- $\beta 1$ activity. J Cell Biol 193: 1305-1319, 2011.

40. Stratman AN, Malotte KM, Mahan RD, Davis MJ and Davis GE: Pericyte recruitment during vasculogenic tube assembly stimulates endothelial basement membrane matrix formation. Blood 114: 5091-5101, 2009.

41. Stratman AN, Schwindt AE, Malotte KM and Davis GE: Endothelial-derived PDGF-BB and HB-EGF coordinately regulate pericyte recruitment during vasculogenic tube assembly and stabilization. Blood 116: 4720-4730, 2010.

42. Yousif LF, Di Russo J and Sorokin L: Laminin isoforms in endothelial and perivascular basement membranes. Cell Adhes Migr 7: 101-110, 2013.

43. Tahajjodi SS, Amerion M, Mahdavi Shahri N, Jalali M and Nikravesh MR: The effect of maternal nicotine on basement membrane collagen IV of brain microvessels changes in neonatal Balb/C mice. Iran J Reprod Med 12: 275-280, 2014.

44. Senger DR and Davis GE: Angiogenesis. Cold Spring Harb Perspect Biol 3: a005090, 2011.

45. Miner JH and Yurchenco PD: Laminin functions in tissue morphogenesis. Annu Rev Cell Dev Biol 20: 255-284, 2004. 